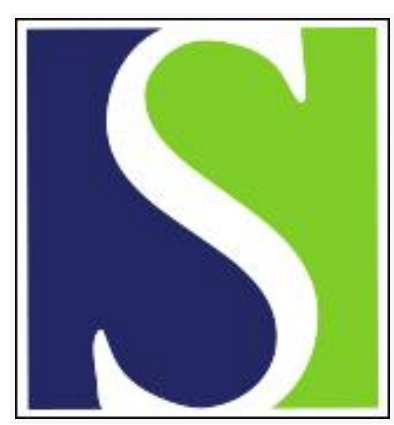

Scand J Work Environ Health 1993;19(4):284-290

https://doi.org/10.5271/sjweh.1472

Issue date: 01 Aug 1993

Electromyographic evaluation of muscular work pattern as a predictor of trapezius myalgia.

by Veiersted KB, Westgaard RH, Andersen P

Affiliation: National Institute of Occupational Health, Department of Physiology, Oslo, Norway.

The following article refers to this text: 2013;39(4):390-400

This article in PubMed: www.ncbi.nlm.nih.gov/pubmed/8235518 


\title{
Electromyographic evaluation of muscular work pattern as a predictor of trapezius myalgia
}

\author{
by Kaj B Veiersted, MD, ${ }^{1}$ Rolf $\mathrm{H}$ Westgaard, $\mathrm{PhD},{ }^{2}$ Per Andersen, $\mathrm{PhD}^{3}$
}

\begin{abstract}
VEIERSTED KB, WESTGAARD RH, ANDERSEN P. Electromyographic evaluation of muscular work pattern as a predictor of trapezius myalgia. Scand $J$ Work Environ Health 1993;19:284-90. Electromyographic (EMG) measurements and interviews concerning muscular pain and disability were performed prospectively every 10 th week on 30 healthy new female employees of a chocolate manufacturing plant. The static muscle activity and rate of short unconscious interruptions in EMG activity (EMG gaps) of the trapezius muscle during repetitive work tasks were evaluated as possible risk factors for patient status with trapezius myalgia. At the start of employment, the static and median contraction levels were significantly higher in future patients than in the rest of the subjects (nonpatients). In subsequent recordings these values were reduced to the level of the nonpatients. Throughout the study, the future patients had a lower frequency of EMG gaps than the nonpatients. A regression analysis showed a significant value for a low rate of EMG gaps to predict future patient status.
\end{abstract}

Key terms: muscle activity pauses, neck and shoulder disorder, prospective design, repetitive work load, static work load.

Occupations with maintained postures and repetitive work tasks are commonly coupled to a high prevalence of neck and shoulder disorders $(1,2)$. Crosssectional studies have shown that patients with trapezius myalgia use their muscles at a higher static level than healthy subjects do (3-5). Subjects who have suffered muscle pain on a previous occasion, but not during a recording session, have also shown a higher static electromyographic (EMG) level than subjects without symptoms (6). Subjects who have experienced muscular pain have demonstrated fewer short, unconscious interruptions in EMG activity (EMG "gaps," with a duration of $0.2-2 \mathrm{~s}$ ) during repetitive work tasks than healthy subjects (6). A previous prospective study of the relation between muscular effort during work and subsequent pain used video recordings as indirect measurements of muscle load (7). It found that relative time with elevated shoulders and a flexed neck was a risk factor.

The present report is the first prospective study with direct measurements of occupational muscular activity and an assessment of muscular pain. The development of muscular pain and subjectively assessed risk factors for trapezius myalgia among workers with repetitive light manual work in the same manufacturing plant have been described elsewhere $(2,8)$.

1 National Institute of Occupational Health, Department of Physiology, Oslo, Norway.

2 The Norwegian Institute of Technology, Division of Organization and Work Science, Trondheim, Norway.

3 Institute of Neurophysiology, University of Oslo, Oslo, Norway.

Reprint requests to: Dr KB Veiersted, National Institute of Occupational Health, PO Box 8149 Dep, N-0033 Oslo, Norway.
The main purpose of this paper was to analyze the association between the level and pattern of muscle usage during the performance of a stereotyped job and the development of trapezius myalgia.

\section{Subjects and methods}

Subjects

Fifty-five female workers hired consecutively by a chocolate manufacturing company were included in the study as they started their employment. Except for three subjects who declined to participate, all of the subjects signed an informed consent form. The study was approved by the regional ethical committee.

The new employees included in the study had not experienced neck or shoulder pain of sufficient severity to seek medical advice for at least one year before employment, and they had no known malformation or illness of the musculoskeletal system in the neck or shoulders. For the inclusion criteria see reference 2 .

\section{Work load exposure}

The subjects were employed full-time, $5 \mathrm{~d}$ a week on the same shift, $61 \%$ of the subjects being on the day shift $(8 \mathrm{~h} / \mathrm{shift})$ and $39 \%$ on the evening shift $(7.5$ $\mathrm{h} /$ shift) with similar work schedules and all on a fixed wage system.

Two different categories of machines were included in the study both on a production line and on a packing line. Of the original 52 persons, a total of 30 subjects remained to be included in the study. (See the inclusion criteria in reference 2.) The EMG measurements made during the work of the dropouts 
showed no significant difference from the remaining subjects. All of the subjects worked with the same category of machine throughout the study. Thirteen subjects $(43 \%)$ worked on the production line with the following three main tasks: feeding the machine with raw materials, repositioning the chocolate bars after the first stage of the process or control of the products and filling or removal of filled trays. The packing work was performed by $17 \mathrm{sub}-$ jects $(57 \%)$ on two different types of machines. Ten subjects worked at a machine with the following three tasks: feeding chocolate from trays, packing wrapped chocolate into boxes, and distributing these boxes. The last task could hardly be said to be machine-paced and had a more dynamic and "lighter" work load. Seven subjects worked on a packing machine which did not have a separate distribution task. The three work tasks were similar for the two categories of machines; therefore it was possible to pool the tasks into feeding (A), repositioning and packing (B), and removal of products from the line (C). Work rotation was performed on all of the machines, with a change of work task every $15-30 \mathrm{~min}$. The work was mainly machine-paced, and the workers had no influence on the speed or organization of the work. The duty cycles were between 20 and $60 \mathrm{~s}$.

\section{Electromyographic measurement of muscular activity level}

The electrical activity of the trapezius muscles during the work of interest was used to evaluate the individual muscular response to the external load. Bipolar surface electrodes were positioned bilaterally and parallel to the muscle fibers, half-way between the acromion and the vertebra prominens. The electrodes had a fixed interelectrode center distance of $2 \mathrm{~cm}$ and were integrated with a preamplifier (AD521, Analog Devices, Norwood, Massachusetts, United States) and placed in a shielded box $(39 \times 20 \times 10 \mathrm{~mm})$. The input impedance was $1 \mathrm{G} \Omega$. Double adhesive tape, with holes for contact gel, was used to fasten the electrodes to the skin.

The EMG signal was bandpass filtered at $10-$ $1300 \mathrm{~Hz}(-3 \mathrm{~dB})$ and analogue-digital converted by a PDP 11/73 computer (Digital Equipment Corporation, Bedford, Massachusetts, United States) at a sample frequency of $2 \mathrm{kHz}$ per channel. The signal was then rectified to full-wave and integrated with a time resolution of $0.2 \mathrm{~s}$.

The contraction levels were determined by the amplitude probability distribution function (APDF) (9). The static level was taken as the contraction level under which the muscle activity was found $10 \%$ of the time and expressed as the percentage of EMG activity of maximal shoulder elevation or arm obduction (MEMG). The median and peak levels were similarly defined as levels under which the activity was found for 50 and $90 \%$ of the time, respectively. The analysis of EMG gaps was introduced in search of patterns suggesting relaxation (6). EMG gaps were defined as periods of at least $0.2 \mathrm{~s}$ of EMG activity below $0.5 \%$ MEMG while the subject worked. They are generally not perceived by the subjects. The total number and the number of gaps longer than $0.6 \mathrm{~s} \cdot \mathrm{min}^{-1}$ were calculated. The mean of the right and left trapezius muscle recordings was used in the analyses. More-detailed information on EMG gaps can be found in reference 6 .

\section{Procedure}

The new employees were asked to join the study within a month after the first day at work. A positive response was followed by a structured interview with a clinical examination and a session with EMG measurement.

Complaints of pain from the neck and shoulder region were recorded continuously in a pain diary, and every 10th week an interview and a clinical examination were performed (2).

The skin was rubbed lightly with ethyl alcohol before application of the EMG electrodes. Before the first EMG recording the electrode position, birthmarks, and bone projections on the neck and shoulders were marked on a transparent plastic sheet to aid accurate repositioning of the electrodes later (11). The first measurement of EMG activity during work was normalized against the electrical response at maximum voluntary force (6). Four submaximal test contractions were also performed before and after the first vocational recording, and for the subsequent recordings every 10th week, to normalize them against the initial measurement. The test contractions lasted $15 \mathrm{~s}$ and were performed with the arms abducted 90 degrees and moved forward 10 degrees from the interaxial line (11). The maximal contractions were only made on the first occasion to avoid risk of muscle injury or postexercise muscle soreness. The time of the vocational EMG recording was adjusted to the habitual work rhythm, and at least 10-min EMG samples were made for all different machine tasks. The subjects were instructed to work as usual. The signal quality, unscheduled work pauses, machine failure, and other special events were noted. The same researcher (KBV) performed all of the interviews and measurements.

The minimum observation time was six months. After termination of the data acquisition, the subjects were divided into a patient $(\mathrm{N}=17)$ and a nonpatient group $(\mathrm{N}=13)$. For the criteria of patient definition see reference 2 . Age, anthropometric data, and shoulder elevation force did not distinguish the patients from the nonpatients (8).

\section{Statistical methods}

The mean of several measurements repeated on a subject was named the "overall mean." The Mann- 
Whitney test was used for the analysis of group medians, the chi-squared test for binomial variables, and the Wilcoxon signed-ranks test for paired samples (12). Analysis of variance for repeated measures with missing values was used for a test of time trend [13 (program 5V)]. Regression analysis was performed with respect to the variable time to event (trapezius myalgia) and time to censorship. The significance of the Kaplan-Meyer plot was tested by a generalized Wilcoxon procedure [13 (program 1L)]. Cox regression with time-dependent covariates was used to determine the hazard ratios [13 (program 2L)]. This ratio indicates the relative risk of reaching patient status when the parameter value of a risk factor (covariate) is increased by one unit (14). The data sets for the survival statistics were constructed with missing data substituted by the preceding nonmissing data. Approximately $10 \%$ of the data was randomly missing. The statistical significance is given by $95 \%$
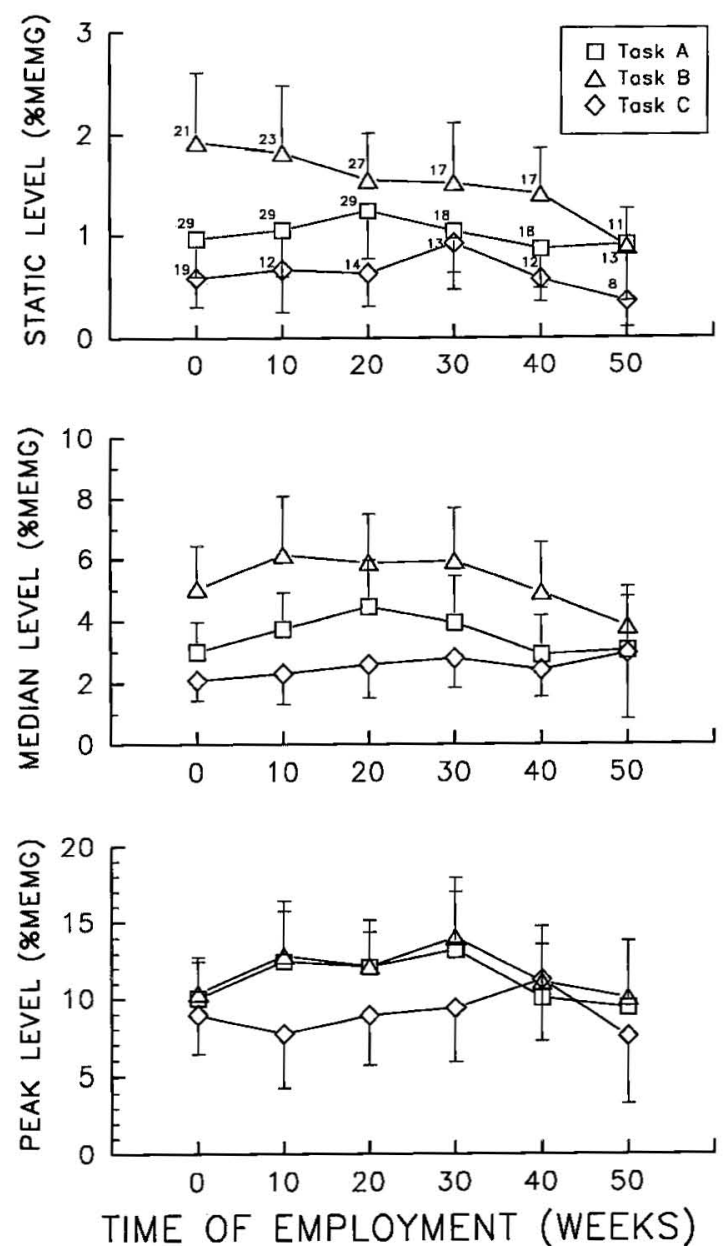

Figure 1. Mean static, median and peak levels of trapezius muscle activity for tasks $A, B$ and $C$ during the first year of employment as a mean of production and packing. The $95 \%$ confidence interval is indicated by bars. The number of subjects underlying each mean value (same for all three panels). (MEMG = maximal electromyographic activity) confidence intervals (95\% CI) and two-tailed P-values less than 0.05 .

\section{Results}

\section{Work tasks and the risk of neck and shoulder pain}

All of the tasks at the various machines represented a low load to the upper trapezius muscles. However, there were distinct differences in the muscle activity, both between the different work tasks (figure 1) and the type of machines (figure 2). Figure 1 shows a decreasing trend for the static level throughout the study, significant for task B. The EMG parameters of task $\mathrm{C}$ were, on the average, considerably lower than for tasks $A$ and $B$. In task $C$ many subjects had a static level below our definition of the resting level ( $0.5 \%$ MEMG). The overall mean of the static levels of tasks A, B, and C for both trapezius muscles during the first year of observation was 1, 1.5, and $0.6 \%$ MEMG, respectively. The corresponding median levels were $3.5,5.3$, and $2.5 \%$ MEMG. The muscle EMG parameters used in the further analyses were the mean values of tasks $A$ and $B$, as these tasks were considered to represent the most significant contribution to the load of the upper trapezius muscles.

The following four EMG parameters were chosen to represent different aspects of the muscular activity pattern: (i) static and (ii) median load levels and the number of (iii) total and (iv) "long" (>0.6 s) EMG gaps per minute (figure 2). The overall contraction level for the packing line (1.5 and 5\% MEMG, static and median loads) was clearly higher than for the production line $(0.9$ and $3.1 \%$ MEMG, static and median loads). The same overall difference was not present for the total number of EMG gaps (9.9 gaps per minute during packing; 13.6 gaps per minute during production), but more long gaps were found during work on the production line (one gap per minute during packing; two gaps per minute during production).

There was no indication that workers on the production line ran a different risk for trapezius myalgia than workers on the packing line. This statement applied whether task $\mathrm{C}$ was part of their work duties or not. This situation is illustrated by the Kaplan-Meyer plot in figure 3, which shows the same cumulative proportion of nonpatients at any time during the first year of employment independent of machine type.

\section{Electromyographic parameters used as risk indicators}

The analysis of EMG parameters for patients and nonpatients was not stratified by machine type, since the risk of contracting patient status was equal for workers on the three machine types (figure 3).

Figure 4 shows the same four EMG parameters as in figure 2 , but now the patients are compared with 


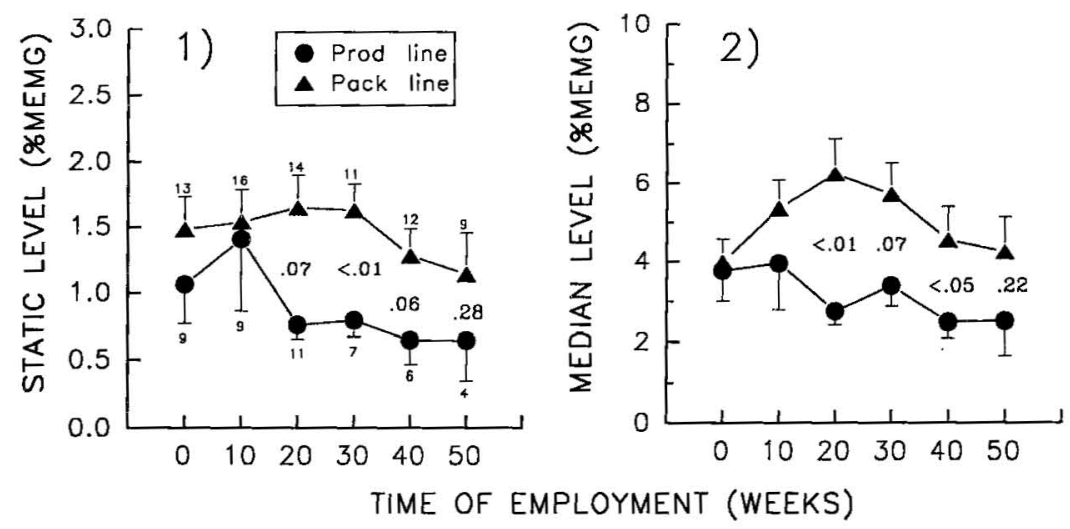

Figure 2. Mean static and median contraction levels and the mean total number and number of long $(>0.6 \mathrm{~s})$ electromyographic (EMG) gaps per minute on the production and the packing lines. The standard error is indicated by bars, and the P-values for Mann-Whitney tests are shown between the means of considerable difference. The number of subjects underlying each mean value (same for all four panels) are also shown in 1).

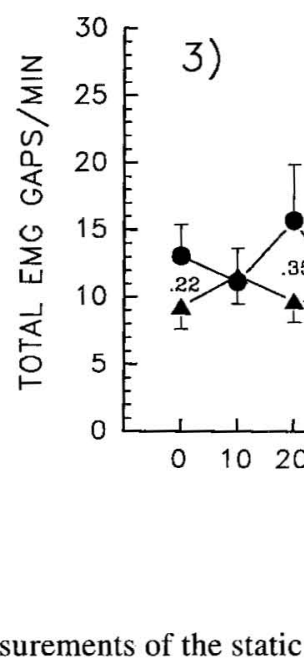

the nonpatients. The first measurements of the static and median EMG levels of the patients were significantly higher than for the nonpatients. This difference was no longer significant after 10 weeks of employment and was not present at all after longer employment times. The static and median EMG levels showed a significantly declining trend for the patient group, but was stable or showed a small increase for the nonpatients. In contrast, the total number of EMG gaps per minute was consistently higher for the nonpatients than for the patients throughout the observation period.

A separate regression analysis was carried out for each of the four EMG load parameters as time-dependent risk factors (table 1). The first measurement of the EMG parameters was analyzed separately from the following measurements up to the time of patient status or to the time of censoring for nonpatients. The first measurement of the static EMG level showed a doubled risk of neck and shoulder complaints if the level increased from 1 to $2 \%$ MEMG. This effect of the static level became insignificant when the subsequent recordings before patient status were used. The median EMG level showed a similar result, though less pronounced. The analysis of all of the EMG gap recordings except the first indicated that the risk of becoming a patient decreased by $6 \%$ for each additional gap per minute.

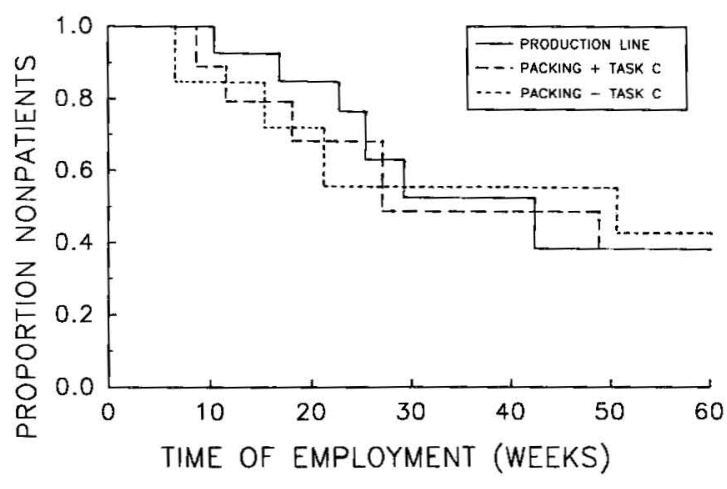

Figure 3. Cumulative proportion of nonpatients on the different lines during the first year of employment (Kaplan-Meyer plot).

This finding was also illustrated by the KaplanMeyer plot in figure 5, where the subjects with fewer gaps per minute showed a significantly higher probability of becoming patients.

\section{Discussion}

The ability of several EMG parameters to predict trapezius myalgia was evaluated in a cohort in which 17 of 30 subjects developed disease within one year 

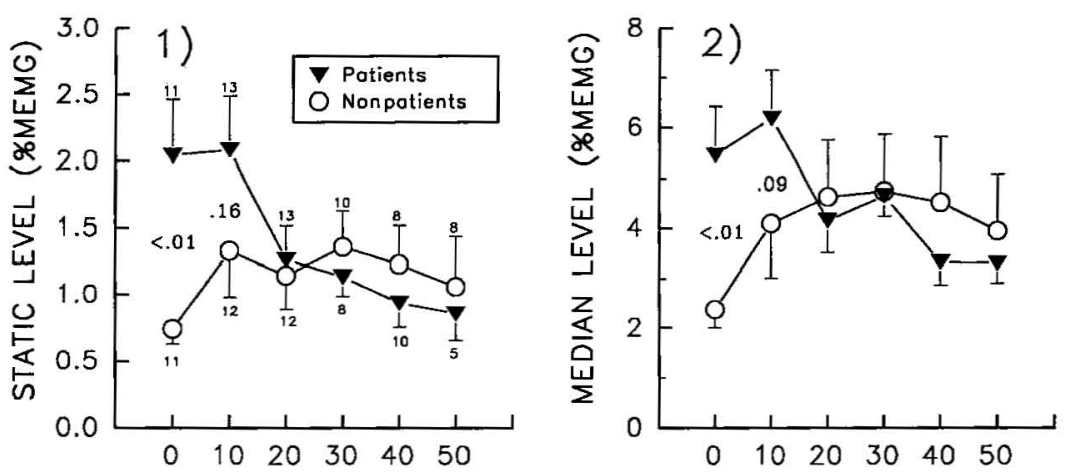

TIME OF EMPLOYMENT (WEEKS)

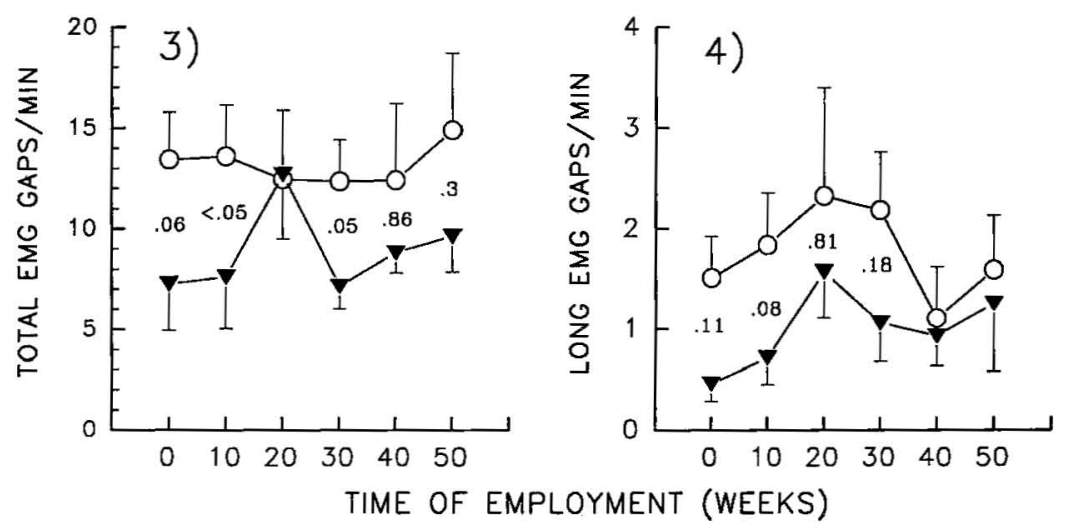

Figure 4. Mean static and median contraction levels and the mean total number and number of long (>0.6 s) EMG gaps per minute for the patients and nonpatients. The standard error is indicated by bars, and the $P$-values for Mann-Whitney tests are shown between the means of considerable difference. The number of subjects underlying each mean value (same for all four panels) are also shown in 1).

Table 1. Hazard ratios for the effect of occupational muscular activity on the development of trapezius myalgia. ( $95 \%$ $\mathrm{Cl}=95 \%$ confidence interval)

\begin{tabular}{|c|c|c|c|c|}
\hline & \multicolumn{2}{|c|}{ First recording ${ }^{2}$} & \multicolumn{2}{|c|}{$\begin{array}{l}\text { Subsequent } \\
\text { recordings }^{a}\end{array}$} \\
\hline & 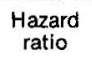 & $95 \% \mathrm{Cl}$ & $\begin{array}{c}\text { Hazard } \\
\text { ratio }\end{array}$ & $95 \% \mathrm{Cl}$ \\
\hline $\begin{array}{l}\text { Static level } \\
\text { Median level } \\
\text { Total gaps/min } \\
\text { Long gaps/min }\end{array}$ & $\begin{array}{l}2.11 \\
1.45 \\
0.94 \\
0.65\end{array}$ & $\begin{array}{r}1.43-3.12 \\
1.2-1.77 \\
0.87-1.01 \\
0.41-1.04\end{array}$ & $\begin{array}{l}1.41 \\
1.12 \\
0.94 \\
0.69\end{array}$ & $\begin{array}{l}0.93-2.13 \\
0.98-1.27 \\
0.89-0.99 \\
0.43-1.12\end{array}$ \\
\hline
\end{tabular}

a First and subsequent recordings have been separated.

in a plant with light manual work. The number of EMG gaps was significantly lower for the patients throughout the study, also before patient status, indicating a possible causal relation between this muscular activity parameter and trapezius myalgia.

\section{Validity of the electromyographic measurements}

Electromyography measures the response to muscle load and may, through a calibration procedure, provide an estimate of this load $(15,16)$. The large interindividual variation in the EMG data when similar tasks are performed is a common observation. It may be due to different work technique $(6,17)$ or

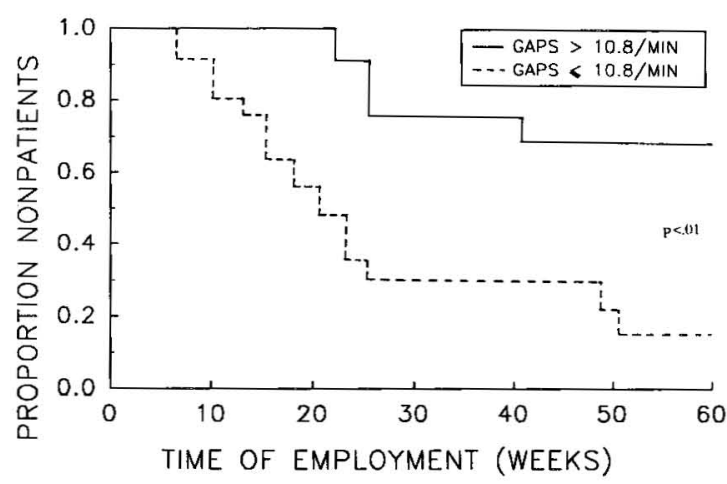

Figure 5. Cumulative proportion of nonpatients in two subgroups with a temporal work pattern above or below 10.8 gaps per minute. This dividing value was the overall mean of all electromyographic (EMG) gap values to the time patient status began or up to the time of censoring for nonpatients, excluding data from the first measurement.

to the generation of excessive muscle force in relation to demands (18). The coefficient of variation was $20 \%$ for the static EMG level and 50\% for the gaps when eight repeated measurements were performed during a week in another study (unpublished data). Consequently, the high intraindividual variability, especially for gaps, demands large group contrasts to detect significant differences. 


\section{Static muscular activity and disorders}

The few prospective studies correlating occupational work load and the development of muscular pain $(7,17,19)$ suggest that static load can be a risk factor. However, in the quoted studies only indirect measures or subjective evaluation of the muscular load was used. Some cross-sectional studies have reported a correlation between symptoms of muscular pain and high static EMG responses evaluated by the APDF method of Jonsson $(5,6,9)$; others have not $(20,21)$.

A possible effect of the initial high static level on the later development of trapezius myalgia was less likely, as the subjects mostly became patients after several months up to one year after the start of employment. This initial higher static level for patients is puzzling. One aspect distinguishing the first EMG recording from the subsequent measurements is the novelty of the employment, as well as the experimental situation, which may have been perceived as stressing. One hypothesis would be that future patients are prone to a higher muscular reactivity to stress compared with those that avoid patient status $(3,18,22)$. However, it must be emphasized that our study was not designed to address the possible reactivity of the patient group.

\section{Temporal pattern of muscular activity}

All muscles need to rest after a period of activity. In an occupational context the duration of a workday and some rest pauses are scheduled by law, collective bargaining, or agreement at the local factory. Shorter, unscheduled pauses are common in most occupations, depending on the type and intensity of the work load. Even during persistent work, the task itself or the individual work technique often makes it possible to interrupt muscle activity to obtain a resting state for some period of time. These periods of rest are often not perceived by the workers and can be very short. However, currently, there are no definite conclusions regarding the effects of duration, distribution, and type of interruption in muscular load on the development of neck and shoulder disorders.

The EMG gaps of the present study have not been considered by other authors, and their physiological significance is unclear. The gaps are interruptions in EMG activity during work, unconscious to the subject, and they are mostly of shorter duration than $0.5 \mathrm{~s}$. Our results showed that patients have fewer EMG gaps than nonpatients, also before contracting trapezius myalgia, during low-level repetitive work. There was no time trend, and therefore it is suggested that the EMG gaps were a stable quality of the temporal activity pattern. The individual ability to achieve these gaps could be augmented by the low static muscular load in this particular plant.
Electromyographic gaps and the pathophysiology of muscular pain

Maintained muscle activation is a factor postulated to be associated with muscular pain, and, if so, it acts through at least three possible mechanisms. Impeded blood flow may be one, though it was less likely in our study, as the contraction levels caused only a fraction of the intramuscular pressure necessary to impede blood flow (23). Yet the unevenly distributed blood flow of many muscles (24) may cause parts of the muscle to be susceptible to small blood flow changes. Second, cell injury due to biochemical changes such as calcium overload of mitochondria (25) may be a basis for membrane defects and visible injury - what is called "moth-eaten fibers" (26). Third, sensitization of nociceptors for originally innocuous stimuli due to, for example, potassium wash out from the continuously activated muscle fibers is another possibility $(27,28)$. The EMG gaps may prove beneficial in this kind of job, by improving blood flow, by allowing calcium to be reabsorbed into the sarcoplasmatic reticulum, or by reducing the extracellular efflux of potassium.

\section{Electromyographic parameters as predictors of muscular pain}

Hägg \& Suurküla (29) found no predictive value of fatigability (decrease in zero crossings of the EMG) of the trapezius muscle during a standardized work session. The static EMG level during work did not predict trapezius myalgia in our study, but this result does not exclude higher static load as a risk factor. However, our material suggests a low rate of EMG gaps as a weak but significant predictor of trapezius myalgia. But this study does not show that EMG gaps is an optimal parameter for characterizing such variability, as alternative quantification methods have not been tested. Yet the EMG gap analysis has qualities consistent with our hypotheses of generic risk factors for the development of trapezius myalgia [ie, prolonged muscle activation due to repetitive work (30), psychosocial problems, and stress $(8,18,31)]$.

\section{Acknowledgments}

This project was supported by grants from the Norwegian Science and Engineering Research Council and from Freia A/S.

We are grateful for assistance from Ms M Bull and Ms B Jarmark.

\section{References}

1. Hagberg M, Wegman DH. Prevalence rates and odds ratios of shoulder-neck diseases in different occupational groups. Br J Ind Med 1987;44:602-10.

2. Veiersted KB, Westgaard RH. Development of trapezius myalgia among female workers performing light manual work. Scand J Work Environ Health 1993; 
19:277-83.

3. Lundervold A. Occupational myalgia: electromyographic investigations. Acta Psychiatr Neurol Scand 1951;26:359-69.

4. Erdelyi A, Sihvonen T, Helin P, Hänninen O. Shoulder strain in keyboard workers and its alleviation by arm supports. Int Arch Occup Environ Health 1988;60: $119-24$

5. Philipson L, Sørbye R, Larsson P, Kaladjev S. Muscular load levels in performing musicians as monitored by quantitative electromyography. Med Probl Performing Artists 1990;5:79--82.

6. Veiersted KB, Westgaard RH, Andersen P. Pattern of muscle activity during stereotyped work and its relation to muscle pain. Int Arch Occup Environ Health 1990;62:31- 41 .

7. Jonsson BG, Persson J, Kilbom $\AA$. Disorders of the cervicobrachial region among female workers in the electronics industry: a two-year follow up. Int J Ind Ergon 1988;3:1-12.

8. Veiersted KB, Westgaard RH. Work-related risk factors for trapezius myalgia. In: Hagberg M, Kilbom $\AA$, ed. International scientific conference on prevention of work-related musculoskeletal disorders PREMUS: book of abstracts, Sweden, 12-14 May 1992. Stockholm: Arbetarskyddsverket, 1992:307-9. (Arbete och hälsa; no 17.)

9. Jonsson B. Kinesiology - with special reference to electromyographic kinesiology. In: Cobb WA, van Duijn $\mathrm{H}$, ed. Contemporary clinical neurophysiology. Amsterdam: Elsevier Scientific Publishing Company, 1978:417-428. (EEG suppl no 34.)

10. Westgaard RH, Jansen T. Individual and work-related factors associated with symptoms of musculoskeletal complaints: I. a quantitative registration system. $\mathrm{Br} \mathrm{J}$ Ind Med 1992;49:147-53.

11. Veiersted KB. The reproducibility of test contractions for calibration of electromyographic measurements. Eur J Appl Physiol 1991;62:91-8.

12. Norusis MJ. SPSS/PC+ V2.0: base manual. Chicago, IL: Statistical Package for Social Sciences Inc, 1988.

13. Dixon WJ. BMDP Statistical software manual. Berkeley, CA: University of California Press, 1990.

14. Cox DR. Regression models and life-tables. J R Stat Soc 1972;34:187-220.

15. Hagberg $M$. Work load and fatigue in repetitive arm elevations. Ergonomics 1981;24:543—55.

16. Antii CJ. Relationship between time means of external load and EMG amplitude in long term myoelectric studies. Electromyogr Clin Neurophysiol 1977;17: $45-53$.

17. Kilbom Å, Persson J. Work technique and its consequences for musculoskeletal disorders. Ergonomics 1987;30:273-9.

18. Westgaard RH, Bjørklund R. Generation of muscle tension additional to postural muscle load. Ergonomics 1987;30:911-23.

19. Leino P, Hasan J, Karppi S-L. Occupational class, physical workload, and musculoskeletal morbidity in the engineering industry. $\mathrm{Br} \mathrm{J}$ Ind Med 1988;45:672 81 .

20. Kuorinka I. EMG analysis of muscle function in repetitive tasks in laboratories and in industry. In: Buser PA, Cobb WA, Okuma T, ed. Kyota symposium: electroencephalography and clinical neurophysiology. Amsterdam: Elsevier Biomedical Press 1982:733-7. (EEG suppl no 36.)

21. Takala E-P, Viikari-Juntura E. Muscular activity in simulated light work among subjects with frequent neck-shoulder pain. Int J Ind Ergon 1991;8:157-64.

22. Elert JE, Rantapää Dahlquist $\mathrm{SB}$, Henriksson-Larsén $\mathrm{K}$, Gerdle $\mathrm{B}$. Increased EMG activity during short pauses in patients with primary fibromyalgia. Scand J Rheumatol 1989;18:321 -3.

23. Järvholm U, Styf J, Suurküla M, Herberts P. Intramuscular pressure and muscle blood flow in suprasinatus. Eur J Appl Physiol 1988;58:219-24.

24. Iversen PO, Standa M, Nikolaysen G. Marked regional heterogeneity in blood flow within a single skeletal muscle at rest and during exercise hyperaemia. Acta Physiol Scand 1989;136:17-28.

25. Edwards RHT. Hypotheses of peripheral and central mechanisms underlying occupational muscle pain and injury. Eur J Appl Physiol 1988;57:275-81.

26. Larsson $\mathrm{S}$, Bengtsson $\mathrm{A}$, Bodegård L, Henriksson KG, Larsson J. Muscle changes in work related chronic myalgia. Acta Orthop Scand 1988;59:552 -6.

27. Sjøgaard G. Muscle energy metabolism and electrolyte shifts during low-level prolonged static contractions in man. Acta Physiol Scand 1988;134:181-7.

28. Dougherty PM, Willis WD. Enhancement of spinothalamic neuron responses to chemical and mechanical stimuli following combined micro-ionophoretic application of N-methyl-D-aspartate acid and substance P. Pain 1991;47:85-93.

29. Hägg GM, Suurküla J. Zero crossing rate of electromyograms during occupational work and endurance tests as predictors for work related myalgia in the shoulder/neck region. Eur J Appl Physiol 1991;62: 436-44.

30. Keyserling WM, Armstrong TJ, Punett L. Ergonomic job analysis: a structured approach for identifying risk factors associated with overexertion injuries and disorders. Appl Occup Environ Hyg 1991;6:353-63.

31. Wærsted M, Eken T, Westgaard RH. Single motor unit activity in psychogenic trapezius muscle tension: a pilot study. In: Hagberg M, Kilbom A, ed. International scientific conference on prevention of work-related musculoskeletal disorders PREMUS: book of abstracts, Sweden, 12-14 May 1992. Stockholm: Arbetarskyddsverket, 1992: 319-21. (Arbete och hälsa; no 17.)

Received for publication: 16 October 1992 